\title{
On the vanishing of principal value integrals
}

\author{
Jan Denef Philippe Jacobs
}

\begin{abstract}
In this article we obtain some results and state a conjecture on the vanishing of principal value integrals over local fields. These integrals were first introduced by Langlands. They appear as the coefficients of the asymptotic expansion of fibre integrals and of oscillating integrals. They also appear as residues of poles of Igusa's local zeta functions. We only give a sketch of the proof of our main result.
\end{abstract}

\section{Rsum}

Dans cet article nous obtenons des rsultats et nous proposons une conjecture concernant la nullit de valeurs principales d'intgrales. Ces valeurs principales ont $t$ introduites par Langlands. Elles apparassent comme coefficients du dvloppement asymptotique d'intgrales dans les fibres et d'intgrales oscillantes. Elles apparassent aussi comme rsidus de ples de fonctions zta locales d'Igusa. Nous donnons seulement une indication de la preuve de notre rsultat principal.

\section{Version franaise abrge:}

\section{Annulation de valeurs principales d'intgrales}

\section{Le cas rel.}

( 1.1 ) Soit $X$ une varit algbrique complexe non singulire projective dfinie sur $\mathbb{R}$ de dimension complexe $m$ tel que $X(\mathbb{R}) \neq \emptyset$. Soit $\omega$ une $m$-forme diffrentielle rationnelle de degr $d$ sur $X$ dfinie sur $\mathbb{R}$. (Donc $\omega \in \Gamma\left(U, \Omega_{X / \mathbb{R}}^{m}{ }^{\otimes d}\right)$ pour un certain ouvert Zariski dense $U \subset X$.) Soit $\operatorname{div}(\omega)=\sum_{i} \beta_{i} D_{i}$. Formellement nous considrons $\omega^{1 / d}$ comme une forme diffrentielle rationnelle multiforme sur $X$ et nous dfinissons $\operatorname{div}\left(\omega^{1 / d}\right):=\sum_{i} \alpha_{i} D_{i}$ o $\alpha_{i}=\beta_{i} / d$. On dit que $\omega^{1 / d}$ n'a pas de ples entiers si les $\alpha_{i}$ ne sont pas des entiers strictement ngatifs.

Supposons maintenant que le support $|\operatorname{div}(\omega)| \operatorname{de} \operatorname{div}(\omega)$ est croisements normaux sur $\mathbb{R}$ (i.e. $\omega$ est croisements normaux et les composantes irrducibles contenant un point $\mathbb{R}$-rationel sont dfinies sur $\mathbb{R}$ ). De plus nous supposons que $\omega^{1 / d}$ n'a pas de ples entiers. Alors on peut dfinir $P V \int_{X(\mathbb{R})}\left|\omega^{1 / d}\right|$, la valeur principale de l'intgrale de $\left|\omega^{1 / d}\right|$ sur $X(\mathbb{R})$ comme dans [7]. Ceci est expliqu dans la dfinition suivante. 
( 1.2 ) Dfinition. Choisissons un nombre fini de coordonnes locales $\left\{x_{p}: U_{p} \rightarrow \mathbb{R}^{m}\right\}_{p \in P}$ pour $X(\mathbb{R})$, centres en $p \in X(\mathbb{R})$, tel que les $U_{p}$ recouvrent $X(\mathbb{R})$ et tel que sur chaque $U_{p}$ on ait $\omega=\xi_{p} x_{p}{ }^{\gamma_{p}}\left(d x_{p}\right)^{d}$, o $\xi_{p}$ est une fonction rgulire sur $U_{p}$, dfinie sur $\mathbb{R}$ et non nulle sur $U_{p}$, et $x_{p}{ }^{\gamma_{p}}=\prod_{i} x_{p_{i}}{ }^{\gamma_{p_{i}}}$ o $\gamma_{p_{i}} \in \mathbb{Z}$. (Ceci est possible parce que $|\operatorname{div}(\omega)|$ est croisements normaux sur $\mathbb{R}$.) ${ }^{\text {Choi- }}$ sissons une partition de l'unit $C^{\infty}\left\{\varphi_{p}\right\}_{p \in P}$ par rapport $\left\{U_{p}\right\}_{p \in P}$. Alors, pour chaque $p \in P$ l'intgrale $\int_{U_{p}} \varphi_{p}\left|\xi_{p}\right|^{1 / d}\left|x_{p}\right|^{\gamma_{p} / d+s}\left|d x_{p}\right|$ converge pour $\operatorname{Re}(s)>>0$ et son prolongement mromorphe est holomorphe en $s=0$ parce que $\omega^{1 / d}$ n'a pas de ples entiers.

Ainsi on peut dfinir $P V \int_{X(\mathbb{R})}\left|\omega^{1 / d}\right|:=\sum_{p \in P^{s \rightarrow 0}} \int_{U_{p}} \varphi_{p}\left|\xi_{p}\right|^{1 / d}\left|x_{p}\right|^{\gamma / d+s}\left|d x_{p}\right|$ o $\lim _{s \rightarrow 0}$ signifie prendre la valeur du prolongement mromorphe de l'intgrale en $s=0$. Cette dfinition est indpendante des choix faits.

( 1.3 ) Soit $\mathcal{L}\left(\omega^{1 / d}\right)$ le faisceau d'espaces vectoriels sur $\mathbb{C}$ localement constant sur $X-|\operatorname{div}(\omega)|$, associ $\omega^{1 / d}$ qui est lisse de rang 1. Une section non-nulle de $\mathcal{L}\left(\omega^{1 / d}\right)$ sur un ouvert connexe $U$ est une branche analytique de $\omega^{1 / d}$ sur $U$ multiplie par un nombre complexe.

(1.4) Thorme Principal. Si $H^{m}\left(X(\mathbb{C})-|\operatorname{div}(\omega)|, \mathcal{L}\left(\omega^{1 / d}\right)\right)=0$ alors

$$
P V \int_{X(\mathbb{R})}\left|\omega^{1 / d}\right|=0
$$

( 1.5 ) Exemple. Soit $X=\mathbb{P}^{1}(\mathbb{C})$ et $U=\mathbb{A}^{1}(\mathbb{C})$ avec coordonne affine $x$. Soit $\omega$ la 1-forme diffrentielle rationnelle de degr $d$ sur $X$, donne sur $U-\{0\}$ par $x^{\beta}(d x)^{d}$. Supposons que $\beta / d \notin \mathbb{Z}$. Alors $|\operatorname{div}(\omega)|=\{0, \infty\},|\operatorname{div}(\omega)|$ est croisements normaux sur $\mathbb{R}, \quad \omega^{1 / d}$ n'a pas de ples entiers et $H^{1}\left(\mathbb{P}^{1}(\mathbb{C})-\{0, \infty\}, \mathcal{L}\left(\omega^{1 / d}\right)\right)=0$. Donc $P V \int_{\mathbb{P}^{1}(\mathbb{R})}\left|\omega^{1 / d}\right|=0$. L'analogue pour des corps locaux non archimdiens de caractristique zro est apparu d'abord dans [8] comme consquence d'un calcul lmentaire.

Pour d'autres exemples, voir (1.1.5).

\section{Le cas p-adique.}

( 2.1 ) Soit $X$ une varit algbrique non singulire projective sur $\mathbb{Q}_{p}$ de dimension $m$. Soit $\omega$ une $m$-forme diffrentielle rationnelle de degr $d$ sur $X$, dfinie sur $\mathbb{Q}_{p}$. Supposons que le support de $\operatorname{div}(\omega)$ est croisements normaux sur $\mathbb{Q}_{p}$ et que les $\alpha_{i}$ sont diffrents de -1 , o les $\alpha_{i}$ sont dfinis comme en (1.1). Alors on dfinit $P V \int_{X\left(\mathbb{Q}_{p}\right)}\left|\omega^{1 / d}\right|$, la valeur principale de l'intgrale de $\left|\omega^{1 / d}\right|$ sur $X\left(\mathbb{Q}_{p}\right)$, de faon analogue (1.2), cf [6]. Choisissons un plongement de $\mathbb{Q}_{p}$ dans $\mathbb{C}$, ce qui donne un sens $X(\mathbb{C})$. Nous proposons la conjecture suivante.

( 2.2 ) Conjecture. Si $H^{i}\left(X(\mathbb{C})-|\operatorname{div}(\omega)|, \mathcal{L}\left(\omega^{1 / d}\right)\right)=0$ pour tout $i \geq 0$ alors

$$
P V \int_{X\left(\mathbb{Q}_{p}\right)}\left|\omega^{1 / d}\right|=0
$$




\section{Statement of the main theorem.}

\subsection{The real case.}

( 1.1.1 ) Let $X$ be a non-singular complex projective algebraic variety defined over $\mathbb{R}$ of complex dimension $m$ such that $X(\mathbb{R}) \neq \emptyset$. Let $\omega$ be a rational differential $m$-form of degree $\mathrm{d}$ on $X$ defined over $\mathbb{R}$. (Thus $\omega \in \Gamma\left(U, \Omega_{X / \mathbb{R}}^{m}{ }^{\otimes d}\right)$ for some Zariski dense open $U \subset X$.) Let $\operatorname{div}(\omega)=\sum_{i} \beta_{i} D_{i}$. We formally consider $\omega^{1 / d}$ as a multivalued rational differential form on $X$ and define $\operatorname{div}\left(\omega^{1 / d}\right)=\sum_{i} \alpha_{i} D_{i}$ where $\alpha_{i}=\beta_{i} / d$. We say that $\omega^{1 / d}$ has no integral poles if no $\alpha_{i}$ is a strictly negative integer and that $\omega^{1 / d}$ has no trivial monodromies if no $\alpha_{i}$ belongs to $\mathbb{Z}$.

From now on we assume that the support $|\operatorname{div}(\omega)| \operatorname{of} \operatorname{div}(\omega)$ has normal crossings over $\mathbb{R}$, meaning that it has normal crossings and that each irreducible component containing an $\mathbb{R}$-rational point is defined over $\mathbb{R}$. Moreover we assume that $\omega^{1 / d}$ has no integral poles. Then we can define $P V \int_{X(\mathbb{R})}\left|\omega^{1 / d}\right|$, the principal value integral of $\left|\omega^{1 / d}\right|$ over $X(\mathbb{R})$ as in Langlands paper [7]. This is done in the following definition.

(1.1.2) Definition. Choose a finite number of local coordinates $\left\{x_{p}: U_{p} \rightarrow \mathbb{R}^{m}\right\}_{p \in P}$ for $X(\mathbb{R})$, centered at $p \in X(\mathbb{R})$, such that the $U_{p}$ cover $X(\mathbb{R})$ and on each $U_{p}: \omega=\xi_{p} x_{p}{ }^{\gamma_{p}}\left(d x_{p}\right)^{d}$, where $\xi_{p}$ is a regular function on $U_{p}$, defined over $\mathbb{R}$ and nowhere zero on $U_{p}$, and $x_{p}{ }^{\gamma_{p}}=\prod_{i} x_{p_{i}}^{\gamma_{p_{i}}}$ where $\gamma_{p_{i}} \in \mathbb{Z}$. (This is possible since $|\operatorname{div}(\omega)|$ has normal crossings over $\mathbb{R}$.) Choose a $C^{\infty}$ partition of unity $\left\{\varphi_{p}\right\}_{p \in P}$ with respect to $\left\{U_{p}\right\}_{p \in P}$. Then for each $p \in P$ : $\int_{U_{p}} \varphi_{p}\left|\xi_{p}\right|^{1 / d}\left|x_{p}\right|^{\gamma_{p} / d+s}\left|d x_{p}\right|$ converges for $\operatorname{Re}(s)>>0$ and its meromorphic continuation is holomorphic in $s=0$ because $\omega^{1 / d}$ has no integral poles. Then one defines $P V \int_{X(\mathbb{R})}\left|\omega^{1 / d}\right|:=\sum_{p \in P} \lim _{s \rightarrow 0} \int_{U_{p}} \varphi_{p}\left|\xi_{p}\right|^{1 / d}\left|x_{p}\right|^{\gamma / d+s}\left|d x_{p}\right|$ where $\lim _{s \rightarrow 0}$ means taking the value in $s=0$ of the meromorphic continuation of the integral. This definition is independent of the choices made.

( 1.1.3 ) Let $\mathcal{L}\left(\omega^{1 / d}\right)$ be the locally constant sheaf of $\mathbb{C}$-vectorspaces on $X-|\operatorname{div}(\omega)|$ associated to $\omega^{1 / d}$, which is locally free of rank 1 . A non-zero section of $\mathcal{L}\left(\omega^{1 / d}\right)$ on a connected open $U$ is an analytic branch of $\omega^{1 / d}$ on $U$ multiplied with a complex number.

( 1.1.4 ) Main Theorem. If $H^{m}\left(X(\mathbb{C})-|\operatorname{div}(\omega)|, \mathcal{L}\left(\omega^{1 / d}\right)\right)=0$ then

$$
P V \int_{X(\mathbb{R})}\left|\omega^{1 / d}\right|=0 .
$$

( 1.1.5 ) Corollary. Assume (1.1.1) and suppose that $X-|\operatorname{div}(\omega)|$ is affine, $\omega^{1 / d}$ has no trivial monodromies and $X(\mathbb{C})-|\operatorname{div}(\omega)|$ has Euler-Poincar characteristic zero. Then $P V \int_{X(\mathbb{R})}\left|\omega^{1 / d}\right|=0$.

Indeed the affineness condition implies that the cohomology is trivial in dimension $>m$, and since there is no trivial monodromy there is no difference between 
compactly supported and ordinary cohomology (see [1, 1.19.1]). Thus Poincar duality yields that the hypothesis of (1.1.4) is satisfied.

\subsection{The p-adic case.}

( 1.2.1 ) Let $X$ be a non-singular projective algebraic variety over $\mathbb{Q}_{p}$ of dimension $m$. Let $\omega$ be a rational differential $m$-form of degree $d$ on $X$, defined over $\mathbb{Q}_{p}$. Suppose that the support of $\operatorname{div}(\omega)$ has normal crossings over $\mathbb{Q}_{p}$ and that the $\alpha_{i} \neq-1$, where the $\alpha_{i}$ are as defined in (1.1.1). Then we can define $P V \int_{X\left(\mathbb{Q}_{p}\right)}\left|\omega^{1 / d}\right|$, the principal value integral of $\left|\omega^{1 / d}\right|$ over $X\left(\mathbb{Q}_{p}\right)$, in complete analogy with (1.1.2), cf $[6,8,9]$. Principal value integrals appear as the coefficients of the asymptotic expansion of fibre integrals and of oscillating integrals. They also appear as residues of poles of Igusa's local zeta functions $[4,2]$. Choose an embedding of $\mathbb{Q}_{p}$ into $\mathbb{C}$, which gives a meaning to $X(\mathbb{C})$. We propose the following conjecture.

( 1.2.2 ) Conjecture. If $H^{i}\left(X(\mathbb{C})-|\operatorname{div}(\omega)|, \mathcal{L}\left(\omega^{1 / d}\right)\right)=0$ for all $i \geq 0$ then

$$
P V \int_{X\left(\mathbb{Q}_{p}\right)}\left|\omega^{1 / d}\right|=0 .
$$

This conjecture is compatible with the calculations in [10]. Perhaps we also have to suppose that $X$ and $\operatorname{div}(\omega)$ have good reduction $\bmod p$ and that the $\alpha_{i} \notin \mathbb{Z}$.

\section{Sketch of the proof of the Main Theorem.}

We only give a sketch of the proof and for simplicity we assume that $\omega^{1 / d}$ has no trivial monodromies. For the general case and for more details we refer to [3] or [5].

\subsection{From local to global in the definition of a PV integral.}

( 2.1.1 ) Proposition. There exists $h \in \mathbb{R}(X)$ and a Zariski open neigbourhood $Y$ of $X(\mathbb{R})$ in $X(\mathbb{C})$ such that

1. $h$ has no poles on $Y$,

2. $h \geq 0$ on $X(\mathbb{R})$,

3. $\left|\operatorname{div}_{0}(h)\right| \cap Y=\left|\operatorname{div}\left(\omega^{1 / d}\right)\right| \cap Y$.

Here $\operatorname{div}_{0}(h)$ means the zero divisor of $h$. From now on we fix a function $h$ and a Zariski open set $Y$ as in this proposition.

\section{( 2.1.2 ) Corollary.}

$$
P V \int_{X(\mathbb{R})}\left|\omega^{1 / d}\right|=\lim _{s \rightarrow 0} \int_{X(\mathbb{R})} h^{s}\left|\omega^{1 / d}\right| .
$$


Here the integral at the righthand side converges for $R e(s)>>0$ and extends to a meromorphic function on $\mathbb{C}$ which is holomorphic in $s=0$. With $\lim _{s \rightarrow 0}$ we mean the value in $s=0$ of this meromorphic continuation.

\subsection{Reducing to integration on a cycle.}

( 2.2.1 ) For our proof we constructed a homology theory associated to an open subspace $W$ of $X$, a family of supports $\theta$ on $W$ and a sheaf $\mathcal{F}$ on $W$. This theory is based on chains of simplices of a fixed triangulation $t$ of $X$ which subtriangulates $X-W$. We will denote $i$-dimensional chains, resp. cycles, boundaries and homology, by $C_{i}^{\theta}(W, \mathcal{F})$, resp. $Z_{i}^{\theta}(W, \mathcal{F}), B_{i}^{\theta}(W, \mathcal{F})$ and $H_{i}^{\theta}(W, \mathcal{F})$.

Under certain conditions (which are always fulfilled in the situations we need) our homology modules are naturally isomorphic to the classical Borel-Moore homology modules. However, since $X$ is compact, we only need a finite number of simplices in our approach. The advantage of this approach is that it avoids tedious problems concerning the convergence of integrals which appear in our proof.

( 2.2.2 ) Definition. If $X$ is a Haussdorf topological space we write $c(X)$,resp. $c l(X)$, for the family of supports on $X$ consisting of all compact ,resp. closed, subsets of $X$. If $W$ is a subspace of $X$ and $\theta$ a family of supports on $X$, let $\theta \cap W=\{K \cap W \mid K \in \theta\}$ and $\theta \mid W=\{K \in \theta \mid K \subset W\}$. Both are families of supports on $W$. If $W$ and $F$ are subspaces of $X$ then we define $\operatorname{fin}(F, W)=c(X-F) \cap W$, which is a family of supports on $W$. Note that for $X$ compact, a closed subset of $W$ belongs to $\operatorname{fin}(F, W)$ if and only if its closure in $X$ is disjoint from $F$. We write $H_{i}^{f i n(F)}(W, \mathcal{F})$ for $H_{i}^{f i n(F, W)}(W, \mathcal{F})$, etc.

( 2.2.3 ) Notation. Let $D=\left|\operatorname{div}\left(\omega^{1 / d}\right)\right|$ and $B=X-Y$. Let $R$ be the ring of meromorphic functions of one complex variable $s$ which are rational in $e^{2 \pi i s}$ without poles in $s \in \mathbb{Z}$. We define the locally constant sheaf $\mathcal{L}\left(h^{s} \omega^{1 / d}\right)$ of $R$-modules on $X-(B \cup D)$ by taking as sections above an open set $U$ products of an analytic branch of $h^{s} \omega^{1 / d}$ on $U$ times an element in $R$. This sheaf is locally free of rank 1.

( 2.2.4 ) For $z$ in $Z_{m}^{f i n(B)}\left(X-(B \cup D), \mathcal{L}\left(h^{s} \omega^{1 / d}\right)\right)$ and $R e(s)>>0$, we define $\int_{z} h^{s} \omega^{1 / d}$ in the obvious way. Because of our approach (2.2.1) one easily verifies that this integral converges to an analytic function of $s$, and only depends on the homology class of the cycle $z$. Similarly we define $\int_{z} \omega^{1 / d}$ for $z$ in $Z_{m}^{f i n(D)}\left(X-D, \mathcal{L}\left(\omega^{1 / d}\right)\right)$

( 2.2.5 ) Proposition. There exists a cycle $z$ in $Z_{m}^{f i n(B)}\left(X-(B \cup D), \mathcal{L}\left(h^{s} \omega^{1 / d}\right)\right)$ such that

$$
\int_{X(\mathbb{R})} h^{s}\left|\omega^{1 / d}\right|=\int_{z} h^{s} \omega^{1 / d}
$$

for $\operatorname{Re}(s)>>0$. 


\subsection{Changing the supports.}

( 2.3.1 ) Theorem. For all $i \geq 0$ we have:

1. The natural morphism

$$
H_{i}^{f i n(D)}\left(X-D, \mathcal{L}\left(\omega^{1 / d}\right)\right) \rightarrow H_{i}^{f i n(\emptyset)}\left(X-D, \mathcal{L}\left(\omega^{1 / d}\right)\right)
$$

is an isomorphism.

2. The natural morphism

$$
H_{i}^{f i n(B \cup D)}\left(X-(B \cup D), \mathcal{L}\left(h^{s} \omega^{1 / d}\right)\right) \rightarrow H_{i}^{f i n(B)}\left(X-(B \cup D), \mathcal{L}\left(h^{s} \omega^{1 / d}\right)\right)
$$

is an isomorphism.

Part 1 follows from a well-known principle [1, prop.1.19] since $\omega^{1 / d}$ has no integral poles. Part 2 is a generalization of part 1 and was one of the difficulties in the proof of the Main Theorem. Remark that $\operatorname{fin}(\emptyset, X-D)=\operatorname{cl}(X-$ $D), \operatorname{fin}(D, X-D)=c(X-D)$ in part 1 and $\operatorname{fin}(B \cup D, X-(B \cup D))=$ $c(X-(B \cup D))$ in part 2 .

( 2.3.2 ) Corollary. There exists a cycle $\eta$ in $Z_{m}^{f i n(B \cup D)}\left(X-(B \cup D), \mathcal{L}\left(h^{s} \omega^{1 / d}\right)\right)$ such that

$$
\int_{z} h^{s} \omega^{1 / d}=\int_{\eta} h^{s} \omega^{1 / d}
$$

for $\operatorname{Re}(s)>>0$. (Note that the righthand side converges to an analytic function of $s$ on $X-A$, where $A$ is the set of poles of $\eta$.)

\subsection{Conclusion of the proof.}

( 2.4.1 ) Proposition. There exists $\eta_{0}$ in $Z_{m}^{f i n(D)}\left(X-D, \mathcal{L}\left(\omega^{1 / d}\right)\right)$ such that

$$
\lim _{s \rightarrow 0} \int_{\eta} h^{s} \omega^{1 / d}=\int_{\eta_{0}} \omega^{1 / d} .
$$

( 2.4.2 ) By part 1 of theorem (2.3.1) and a Poincar Duality Theorem we have $H_{m}^{f i n(D)}\left(X-D, \mathcal{L}\left(\omega^{1 / d}\right)\right) \cong H_{m}^{c l(X-D)}\left(X-D, \mathcal{L}\left(\omega^{1 / d}\right)\right) \cong H^{m}\left(X-D, \mathcal{L}\left(\omega^{1 / d}\right)\right)$.

Our assumption that $H^{m}\left(X-D, \mathcal{L}\left(\omega^{1 / d}\right)\right)=0$ then implies that $\eta_{0}$ is a boundary and hence, by a Stokes theorem, $\int_{\eta_{0}} \omega^{1 / d}=0$. Combining the formulas in (2.1.2), (2.2.5), (2.3.2) and (2.4.1) directly yields our Main Theorem (1.1.4).

\section{References}

[1] P.DELIGNE, Sommes trigonomtriques , published in: Cohomologie Etale (SGA $4 \frac{1}{2}$ ), Springer Lecture Notes in Math., 569(1977),168-232. 
[2] J.DENEF, Report on Igusa's local zeta function, Sminaire Bourbaki, 741(1990-91), Astrisque 201/202/203 (1991),359-386.

[3] J.DENEF and P.JACOBS, A cohomological condition for the vanishing of principal value integrals, in preparation.

[4] J.IGUSA, Some aspects of the arithmetic theory of polynomials, published in: Discrete Groups in Geometry and Analysis, Progress in Math. 67, Birkhaser(1987),20-47.

[5] P.JACOBS, Ph.D. thesis, in preparation.

[6] R.P.LANGLANDS, Orbital integrals on forms of Sl(3),I, Amer.J.Math. 105(1983),465-506.

[7] R.P.LANGLANDS, Remarks on Igusa theory and real orbital integrals, published in The Zeta Functions of Picard Modular Surfaces, Les Publications CRM, Montral(1992), distributed by AMS,335-347.

[8] R.P.LANGLANDS and D.SHELSTAD, On principal values on p-adic manifolds, Springer Lecture Notes in Math., 1041(1984),250-279.

[9] R.P.LANGLANDS and D.SHELSTAD, Orbital integrals on forms of Sl(3),II, Can.J.Math. 41(1989),480-507.

[10] W.VEYS, Poles of Igusa's local zeta function and monodromy, Bull. Soc. Math. France 121(1993),545-598.

Jan Denef

University of Leuven

Department of Mathematics

Celestijnenlaan $200 \mathrm{~B}$

B-3001 Leuven

Belgium

e-mail:Jan.Denef@wis.kuleuven.ac.be
Philippe Jacobs

University of Leuven

Department of Mathematics

Celestijnenlaan 200 B

B-3001 Leuven

Belgium

e-mail:Philippe.Jacobs@wis.kuleuven.ac.be 